\title{
Prostate cancer detection by prostate-specific antigen-based screening in the Japanese Hiroshima area shows early stage, low-grade, and low rate of cancer-specific death compared with clinical detection
}

\author{
Jun Teishima, MD, PhD; $;^{*}$ Satoshi Maruyama, MD, PhD; Hideki Mochizuki, MD, PhD; ${ }^{\dagger}$ Kiyotaka Oka, MD;* \\ Kenichiro Ikeda, MD,* Keisuke Goto, MD,* Hirotaka Nagamatsu, MD,* Keisuke Hieda, MD,* Koichi Shoji, MD;* \\ Akio Matsubara, MD, PhD*
}

*Department of Urology, Integrated Health Sciences, Institute of Biomedical and Health Sciences, Hiroshima University, Hiroshima, Japan; 'Department of Urology, Hiroshima General Hospital, Hatsukaichi, Japan; §Department of Urology, Miyoshi Prefectural Hospital, Miyoshi, Japan

Cite as: Can Urol Assoc J 2014;8(5-6):e327-32. http://dx.doi.org/10.5489/cuaj.1715 Published online May 21, 2014.

\section{Abstract}

Introduction: We investigate the effectiveness of prostate-specific antigen (PSA) screening for prostate cancer. We compare the characteristics of 2 sets of patients: (1) those in whom prostate cancer was detected via PSA screening (the PS group) and (2) those in whom prostate cancer was detected at the outpatient office (the non-PS group).

Methods: Between 2002 and 2010, prostate cancer was detected in 315 patients by PSA screening. Their age, initial PSA level, pathological findings in biopsy specimens, clinical stage, and prognosis were compared with those of 497 prostate cancer patients diagnosed at the outpatient office of the Department of Urology, Hiroshima University, in the same period.

Results: The rates of patients with initial PSA higher than $50 \mathrm{ng} /$ $\mathrm{mL}$, with a Gleason score of 8 or higher, and with clinical stage D were significantly lower in the PS group than those in the non-PS group. The 5-year overall survival and cancer-specific survival in the PS group was $91.3 \%$ and $98.2 \%$, respectively; these results were significantly better than those in the non-PS group $(86.4 \%$, $p=0.0178$, and $94.9 \%, p=0.0112$, respectively). A Cox hazard analysis showed that PSA screening was an independent predictive factor for cancer-specific survival.

Conclusions: Although our study is limited by its retrospective nature and small size, the present data indicate that prostate cancer detected in the PS group showed earlier stage, lower grade, and better prognosis than in the non-PS group.

\section{Introduction}

In the United States, prostate cancer has the highest incidence of all malignant tumours and the second highest mortality rate. ${ }^{1}$ Prostate-specific antigen (PSA)-based screening is widely used to prevent death due to prostate cancer. In men over $50,75 \%$ of them have had their PSA levels measured at least once and half of them have their levels measured annually. ${ }^{2}$ While both the incidence and mortality rate of prostate cancer in Japan continue to rise, its mortality rate in the United States has significantly decreased due to high rates of PSA screening. ${ }^{3}$ Although the Japanese Urological Association PSA screening guidelines in 2008 recommend screening in men aged 50 and over, the exposure rate of PSA screening in Japan is still much lower than in the United States. ${ }^{3}$ To determine whether PSA screening decreases prostate cancer death in Japan, it is essential to demonstrate that PSA screening can improve the chance of survival of prostate cancer patients. Therefore, we investigated whether PSA screening alone (without clinical symptoms) can decrease the mortality rate in Japan. To do this, we compared the outcomes of patients who underwent PSA screening with those of the outpatients.

\section{Methods}

Between 2002 and 2010, prostate cancer screening by PSA measurements was performed for men 50 and over in the northern area of Hiroshima Prefecture, Japan. In all screenings, serum PSA levels were measured using the E-test Tosoh II PA assay (Tosoh, Tokyo, Japan). Patients who had abnormal PSA levels were recommended to visit urologists and to undergo further examinations, such as a digital rectal examination (DRE) or a transrectal ultrasound (TRUS). TRUSguided systematic 10-cores prostate needle biopsies were performed via the transrectal approach if necessary.

The thresholds of the serum PSA levels were set according to previously reported age-specific reference ranges of PSA. ${ }^{4}$ These were $3.0 \mathrm{ng} / \mathrm{mL}$ for men between 50-64, $3.5 \mathrm{ng} / \mathrm{mL}$ for men between 65-69, and $4.0 \mathrm{ng} / \mathrm{mL}$ for men 70 and over.

For this 9-year study period, 334 cases of prostate cancer were detected by PSA screening in Hiroshima Prefecture in Japan and designated as the "PS group," and in the same 
period, 500 outpatients were diagnosed with prostate cancer in Hiroshima University Hospital and were designated as the "non-PS group." No patient was counted in both groups. We excluded 19 patients in the PS group and 3 in the non-PS group due to a lack of information. Ultimately, 315 cases in the PS group and 497 cases in the non-PS group were included in this study. In the non-PS group, 304 cases $(61.2 \%)$ had symptomatic diseases at their first outpatient office visit. The mean and median follow-up times of both groups were 47.0 and 42.6 months, respectively.

Age, initial PSA level, pathological diagnosis at biopsy, clinical stage, and prognosis were compared between the PS and the non-PS groups.

\section{Statistical analysis}

Overall survival (OS), cancer-specific survival (CSS), and biochemical relapse-free survival (bRFS) were determined by the Kaplan-Meier method and analyzed by log-rank testing. Predictive factors for CSS were analyzed using a Cox hazard analysis. Each parameter in the PS and non-PS groups was compared using the Mann-Whitney U-test and chi-squared test. All statistical analyses were conducted using a StatView 5.0 software package (SAS Institute, Cary, NC). A $p$ value of less than 0.05 was considered statistically significant.

\begin{tabular}{|c|c|c|c|}
\hline & PS group (\%) & Non-PS group (\%) & $p$ value \\
\hline & $(n=315)$ & $(n=497)$ & \\
\hline \multicolumn{4}{|l|}{ Age } \\
\hline$\geq 80$ & $70(22.2)$ & $47(9.5)$ & $<0.0001$ \\
\hline $70-79$ & $151(48.0)$ & $249(50.1)$ & 0.5478 \\
\hline $60-69$ & $79(25.1)$ & $183(36.8)$ & 0.0005 \\
\hline$\leq 59$ & $15(4.8)$ & $18(3.6)$ & 0.4227 \\
\hline \multicolumn{4}{|c|}{ Initial PSA (ng/mL) } \\
\hline$\geq 50.1$ & $24(7.6)$ & 79 (15.9) & 0.0006 \\
\hline $20.1-50$ & $48(15.2)$ & $60(12.1)$ & 0.1955 \\
\hline $10.1-20$ & $72(22.9)$ & $132(26.6)$ & 0.2359 \\
\hline 4.1-10 & $150(47.6)$ & $209(42.1)$ & 0.1196 \\
\hline$\leq 4$ & $21(6.7)$ & $17(3.4)$ & 0.0328 \\
\hline \multicolumn{4}{|c|}{ Gleason score } \\
\hline$\geq 8$ & $66(21.0)$ & $174(35.0)$ & $<0.0001$ \\
\hline 7 & $145(46.0)$ & $207(41.6)$ & 0.2195 \\
\hline$\leq 6$ & $104(33.0)$ & $116(23.3)$ & 0.0025 \\
\hline \multicolumn{4}{|c|}{ Clinical stage } \\
\hline$B$ & $262(83.2)$ & $393(79.0)$ & 0.1494 \\
\hline C & $44(14.0)$ & $42(8.5)$ & 0.0128 \\
\hline D & $9(2.8)$ & $62(12.5)$ & $<0.0001$ \\
\hline
\end{tabular}

PSA: prostate-specific antigen; PS group: Patients with prostate cancer detected by PSAbased screening in Hiroshima Prefecture. Non-PS group: Patients with prostate cancer diagnosed at the outpatient office at the Hiroshima University Hospital.

\section{Results}

\section{Patient demographics}

The average age of the PS group was 74.3 years old, significantly older than that of the non-PS group (71.0) $(p<0.0001)$ (Table 1). The number of patients over 80 in the PS group was significantly higher than those in the nonPS group ( $p<0.0001)$. Although there was no significant difference in the initial PSA (iPSA) between the PS group (1.13-18740 (median: 11.0) $\mathrm{ng} / \mathrm{mL}$ ) and the non-PS group (2.3-923.3 (median: 9.5$) \mathrm{ng} / \mathrm{mL}$ ), the rate of patients whose iPSA was higher than $50 \mathrm{ng} / \mathrm{mL}$ was significantly lower in the PS group. The distribution of Gleason scores at biopsy was significantly different. The rate of patients with 6 or lower was significantly higher in the PS group; in addition, the rate of patients with 8 or higher was significantly lower in the PS group. The rate of patients with clinical stage D in the PS group was significantly lower than in the non-PS group.

In patients with stage $B$, while the percentage of radiation therapy as an initial treatment in the PS group was significantly lower, the percentage of androgen deprivation therapy in the PS group was significantly higher than in the non-PS group (Table 2). This distribution was reflected in all patients. In contrast, in patients with stage $\mathrm{C}$, while the percentage of radiation therapy as an initial treatment in the PS group was significantly higher, the percentage of androgen

Table 2. Destribution of initial treatments in every stage

\begin{tabular}{lccc} 
& PS group & Non-PS group & $p$ value \\
Stage B & 262 & 393 & \\
RP & $98(37.4)$ & $143(36.4)$ & 0.7913 \\
RT & $21(8.0)$ & $164(41.7)$ & $<0.0001$ \\
ADT & $142(54.2)$ & $79(20.1)$ & $<0.0001$ \\
AS & $1(0.4)$ & $7(1.8)$ & 0.1102 \\
Stage C & 44 & 42 & \\
RP & $3(6.8)$ & $3(7.1)$ & 0.9529 \\
RT & $32(72.7)$ & $18(42.9)$ & 0.0050 \\
ADT & $9(20.5)$ & $21(50.0)$ & 0.0041 \\
Stage D & 9 & 62 & \\
RP & $0(0.0)$ & $1(1.6)$ & 0.7012 \\
RT & $0(0.0)$ & $0(0.0)$ & \\
ADT & $9(100.0)$ & $61(98.4)$ & 0.7012 \\
Total & 315 & 497 & \\
RP & $101(32.1)$ & $147(29.6)$ & 0.4536 \\
RT & $53(16.8)$ & $185(37.2)$ & $<0.0001$ \\
ADT & $160(50.8)$ & $158(31.8)$ & $<0.0001$ \\
AS & $1(0.3)$ & $7(1.4)$ & 0.1251 \\
\hline PSA: prostate-specific antigen; RP: radical prostatectomy; RT: radiation therapy; ADT: \\
androgen deprivation therapy; AS: active surveillance; PS group: Patients with prostate \\
cancer detected by PSA-based screening in Hiroshima Prefecture. Non-PS group: Patients \\
with prostate cancer diagnosed at the outpatient office at the Hiroshima University Hospital. \\
\hline
\end{tabular}




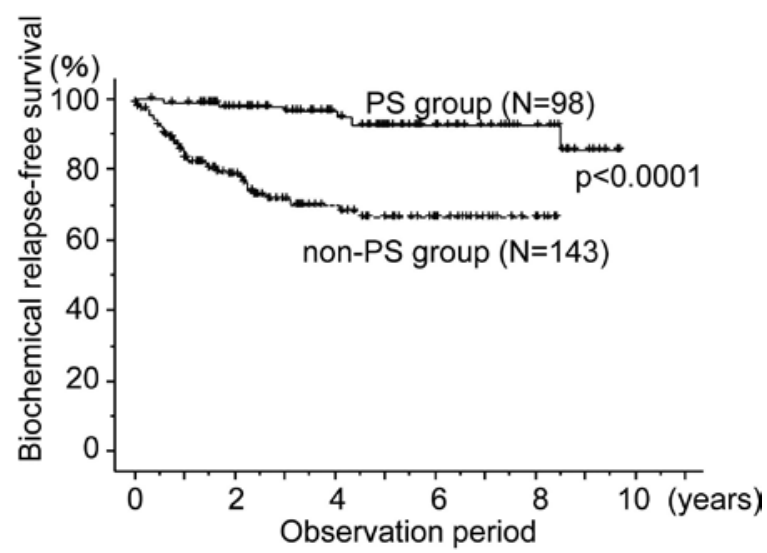

No. of patients at risk

$\begin{array}{lrrrrr}\text { PS group } & 98 & 78 & 53 & 30 & 16 \\ \text { non-PS group } & 143 & 74 & 39 & 23 & 6\end{array}$

Fig. 1. Biochemical-relapse free survival in patients who underwent radical prostatectomy due to stage B prostate cancer. PSA: prostate-specific antigen; PS group: Patients with prostate cancer detected by PSA-based screening in Hiroshima Prefecture. Non-PS group: Patients with prostate cancer diagnosed by outpatient office in Hiroshima University Hospital. The threshold of PSA level for biochemical recurrence after surgery was defined at $0.2 \mathrm{ng} / \mathrm{mL}$ or higher.

deprivation therapy in the PS group was significantly lower than in the non-PS group.

We tallied patients diagnosed as clinical stage $B$ as per the D'Amico risk classification (Table 3). Among these patients, 98 cases in the PS group and 141 cases in the non-PS group underwent radical prostatectomy. Among these patients, the rate of low-risk patients was significantly higher and that of high-risk patients was significantly lower in the PS group compared to the non-PS group. Furthermore, the 5-year bRFS rate was $91.7 \%$ in the PS group, significantly better than in the non-PS group (66.1\%, $p<0.0001)$ (Fig. 1).

OS and CSS in the PS group and the non-PS group were determined by the Kaplan-Meier method (Fig. 2). There was no significant difference between the 5-year CSS of the PS group and that of the non-PS group in each clinical stage $(98.9 \%$ and $98.9 \%, p=0.7613$, in stage $\mathrm{B} ; 96.2 \%$ and $100 \%, p=0.9944$, in stage $C$; and $83.3 \%$ and $59.8 \%$, in stage $D$, respectively), nor was there any significant difference between the 5-year OS of the PS group and the non-PS group in each clinical stage $(91.3 \%$ and $92.1 \%$,
Table 3. D'Amico risk classification in patients with clinical stage $B$ in every treatment option

\begin{tabular}{lccc}
\hline & PS group & Non-PS group & p value \\
\hline RP & 98 & 141 & \\
High (\%) & $13(13.3)$ & $49(34.8)$ & 0.0002 \\
Intermediate (\%) & $53(54.1)$ & $69(48.9)$ & 0.4338 \\
Low (\%) & $32(32.7)$ & $23(16.3)$ & 0.0026 \\
RT & 21 & 164 & \\
High (\%) & $8(38.1)$ & $44(26.8)$ & 0.2796 \\
Intermediate (\%) & $10(47.6)$ & $75(45.7)$ & 0.7022 \\
Low (\%) & $3(14.3)$ & $45(27.4)$ & 0.1954 \\
ADT & 142 & 79 & \\
High (\%) & $46(32.4)$ & $31(39.2)$ & 0.3060 \\
Intermediate (\%) & $67(47.2)$ & $43(54.4)$ & 0.8669 \\
Low (\%) & $29(20.4)$ & $5(6.4)$ & 0.0054 \\
AS & 1 & 7 & \\
High (\%) & $0(0.0)$ & $0(0.0)$ & - \\
Intermediate (\%) & $0(0.0)$ & $1(14.3)$ & 0.6862 \\
Low (\%) & $1(100.0)$ & $6(85.7)$ & 0.6862 \\
Total & 262 & 391 & \\
High (\%) & $67(25.6)$ & $124(31.7)$ & 0.0909 \\
Intermediate (\%) & $130(49.6)$ & $188(48.1)$ & 0.7002 \\
Low (\%) & $65(24.8)$ & $79(20.2)$ & 0.1642 \\
\hline PSA & & & \\
\hline
\end{tabular}

PSA: prostate-specific antigen; RP: radical prostatectomy; RT: radiation therapy; ADT: androgen deprivation therapy; AS: active surveillance; PS group: Patients with prostate cancer detected by PSA-based screening in Hiroshima Prefecture. Non-PS group: Patients with prostate cancer diagnosed at the outpatient office at the Hiroshima University Hospital.

$p=0.8275$ in stage $B$ and $62.5 \%$ and $47.9 \%, p=0.3997$, in stage $D$, respectively), except in stage $C(96.2 \%$ and $84.9 \%$, $p=0.0247)$. In all patients, the 5-year OS and CSS of the PS group were $91.3 \%$ and $98.2 \%$, respectively, significantly better than those of the non-PS group $(86.4 \%, p=0.0178$ and $94.9 \%, p=0.0112$, respectively) (Fig. 2). The multivariate analysis demonstrated that the PSA group was an independent predictive factor for CSS (Table 4).

\section{Discussion}

In this study, we found differences in the characteristics and prognosis between the PS group and the non-PS group of prostate cancer patients in the PSA era in Japan.

The European Randomized Study of Screening for Prostate Cancer (ERSPC) has shown the effect of PSA screening on the

\begin{tabular}{|c|c|c|c|c|}
\hline & \multirow{2}{*}{$\begin{array}{c}\text { Univariate analysis } \\
p \text { value (log-rank) }\end{array}$} & \multicolumn{3}{|c|}{ Multivariate analysis } \\
\hline & & HR & $95 \% \mathrm{Cl}$ & $p$ value \\
\hline PS group vs. non-PS group & 0.0112 & 3.505 & $1.302-9.438$ & 0.0131 \\
\hline Age: $<70$ vs. $\geq 70$ & 0.1000 & 1.650 & $0.611-4.458$ & 0.3229 \\
\hline Initial PSA (ng/mL) $<10$ vs. $10 \geq$ & $<0.0001$ & 10.131 & $2.386-43.022$ & 0.0017 \\
\hline Treatment: prostatectomy vs. others & 0.0026 & 7.500 & $2.062-7.370$ & 0.0502 \\
\hline
\end{tabular}

PSA: prostate-specific antigen; HR: hazard ratio; Cl: confidence interval; PS group: Patients with prostate cancer detected by PSA-based screening in Hiroshima Prefecture. Non-PS group: Patients with prostate cancer diagnosed at the outpatient office at the Hiroshima University Hospital. 
Teishima et al.

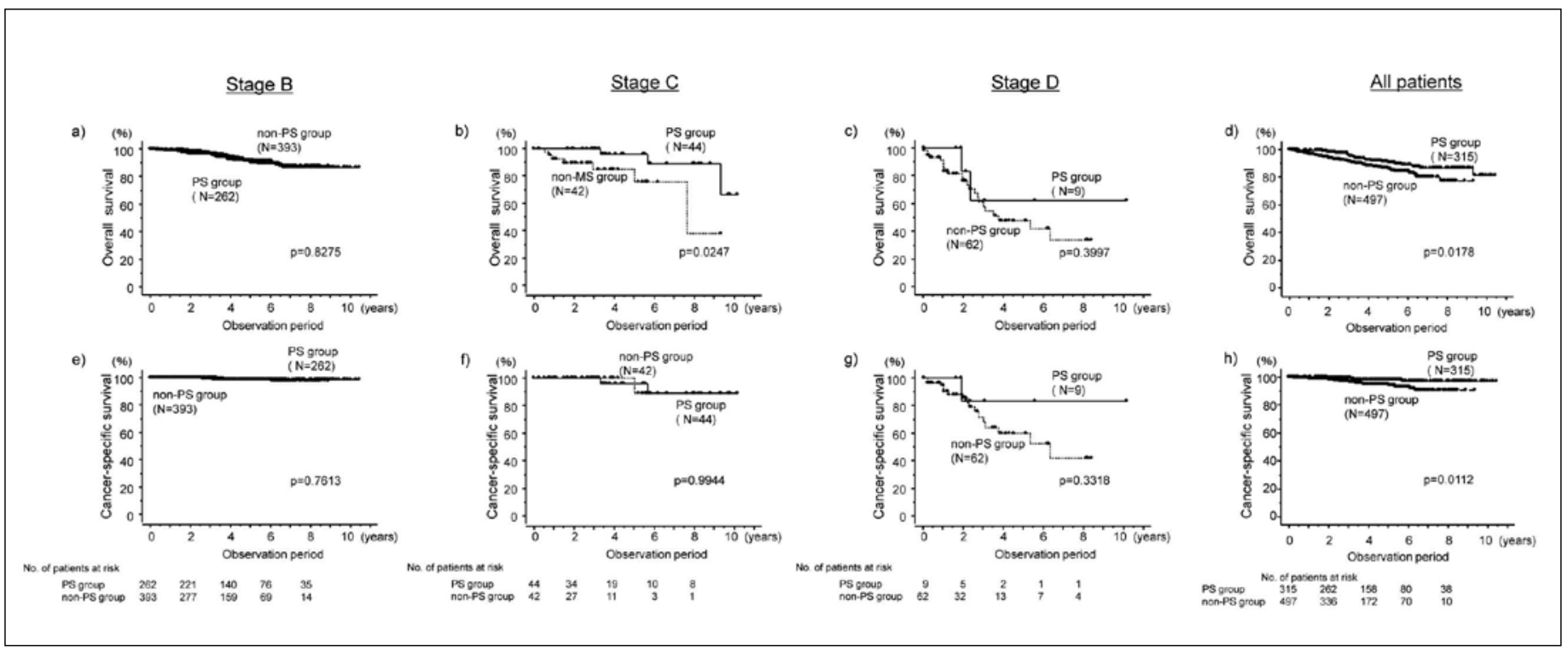

Fig. 2. Overall survival and cancer-specific survival.

reduction of mortality from prostate cancer. ${ }^{5-7}$ Investigators have demonstrated a reduction in prostate cancer mortality due to PSA screening, and advanced studies have reported the risk predicting prostate cancer using PSA based on data from the ERSPC..$^{8-10}$ On the other hand, the Prostate, Lung, Colorectal, and Ovary (PLCO) screening trial showed no benefit of organized population-based annual screening on mortality. ${ }^{11,12}$ In addition to this discrepancy, the incidence of PSA testing for outpatients has increased in several countries even though there are no present prostate cancer symptoms. ${ }^{13-15}$ This is also evident in Japan, which suggests that early stage prostate cancer may be detected in outpatients as well as with PSA screenings. Therefore, it is critical to compare patients with prostate cancer detected by PSA screening and those diagnosed in outpatient offices to assess the importance of PSA screening in Japan.

As mentioned earlier, we categorized prostate cancer patients into 2 groups: (1) the PS group and the non-PS group. The non-PS group included men who were diagnosed with prostate cancer in outpatient offices and thus includes those who underwent PSA testing regardless of the symptoms related to the prostate. In the PS group, there were fewer patients with high Gleason scores, high PSA, and metastatic diseases (Table 1). Previous studies in Gunma Prefecture and Sweden reported that mass screening could decrease the proportion of metastatic prostate cancer. ${ }^{16,17}$ Our data are consistent with those in these previous studies and demonstrate the effectiveness of prostate cancer screening for early detection of prostate cancer. Other reports have demonstrated that PS decreased not only the rate of metastatic diseases, but also the risk of developing them during the follow-up period..$^{18,19}$ Further observation is required to elucidate such effects in our prostate cancer screening.
In our study, in patients with clinical stage B who were treated with radical prostatectomy, the distribution of D'Amico risk classification in the PS group shifted lower compared with that in the non-PS group (Table 3 ). Furthermore, bRFS in the PS group was significantly better than that in the non-PS group (Fig. 1). These data indicate that PSA screening can improve the oncologic outcome of prostate cancer in stage B (Fig. 2, parts A, B, C, E, F and G).

To demonstrate the effectiveness of PSA screening for prostate cancer, one of the most crucial points is to show the decrease of mortality. While neither OS nor CSS in the PS group was significantly better that those of the non-PS group in each clinical stage (except OS in stage C), both the OS and CSS of the PS group were significantly better than those of the non-PS group in all patients (Fig. 2, parts D and H), suggesting that fewer patients with a higher stage might lead to a better prognosis in the PS group. Previous studies have demonstrated that longer survival is expected in patients with prostate cancer detected by mass screening. ${ }^{16}$ In addition to this evidence, our data indicate that PSA screening might improve the prognosis of prostate cancer patients in the PSA era, in which PSA testing can be performed on all older outpatients even if they do not exhibit any symptoms. Despite the fact that the average age of the PS group was older than the non-PS group, better life expectancy was shown in the PS group. This suggests that PSA screening improves the prognosis in prostate cancer patients.

The difference of distribution of the initial treatments for patients with stage B between the PS group and the non-PS group is a limitation of our study. The ratio of radiation therapy as an initial treatment in the PS-group was significantly higher than that in the non-PS group in all patients and in patients with stage B (Table 2). The reason for this distribu- 
tion is that the non-PS group with stage B included patients who underwent brachytherapy using iodine-125 seed, while the PS group did not. Patients in PS group can undergo external beam radiation therapy (EBRT) at the local hospital, while brachytherapy has often been performed for non-PS group diagnosed in the University Hospital in this study. Moreover, the ratio of androgen deprivation therapy as an initial treatment in the PS group was significantly higher than that in the non-PS group. We speculate that the difference of age distribution between the 2 groups was reflected in the choice of the treatment. Specifically, because the PS group included a higher rate of older patients, a higher rate of patients might undergo androgen deprivation therapy. Although older patients with low-risk and localized prostate cancer should undergo active surveillance rather than androgen deprivation therapy, there were few patients who actually underwent it. We should also point out that in addition to the benefits of PSA screening for prostate cancer, there are also some disadvantages. One is unnecessary biopsies and related complications. Data from ERSPC trials showed that $76 \%$ of prostate biopsies for an elevated PSA level detected no cancer tissues. Moreover, 3.5\% developed a fever, $0.4 \%$ experienced urinary retention, and $0.5 \%$ required hospitalization for symptoms of urosepsis. ${ }^{20}$ Another disadvantage is the possibility of unnecessary treatment due to increased diagnosis of early-stage prostate cancer and an increased risk of related complications. Previous randomized control trials have revealed that prostatectomy and radiation therapy are associated with an increased risk for erectile dysfunction and urinary incontinence compared with active surveillance. ${ }^{21,22}$ Insufficient information about treatment options for patients with prostate cancer is one of the most crucial issues in this area and needs to be solved as soon as possible.

\section{Conclusion}

We demonstrated that prostate cancer with an earlier stage, lower grade, and better prognosis was shown in PSA screening. Our results indicate that screening in the Hiroshima area should help improve both the OS and CSS of prostate cancer patients through an expected increase of early detection and decrease of high-stage diseases compared with patients whose cancers are detected in outpatient offices. We consider these results to be crucial terms of promoting PSA screening for prostate cancer. However, the present study was retrospective, and the sample size was too small. Additional accumulation of data obtained from more widely spread screening or the prospective study with high volume is required.

Competing interests: Dr. Teishima, Dr. Maruyama, Dr. Mochizuki, Dr. Oka, Dr. Ikeda, Dr. Goto, Dr. Nagamatsu, Dr. Hieda, Dr. Shoji and Dr. Matsubara all declare no competing financial or personal interests.
This paper has been peer-reviewed.

\section{References}

1. Siegel R, Naishadham D, Jemal A. Cancer statistics. 2012. CA Cancer J Clin 2012;62:10-29. http:// dx.doi.org/10.3322/caac.20138

2. Sirovich BE, Schwartz $L M$, Woloshin $S$. Screening men for prostate and colorectal cancer in the United States: does practice reflect the evidence? JAMA 2003;289:1414-20. http://dx.doi.org/10.1001/ jama.289.11.1414

3. Ito K. Prostate-specific antigen-based screening for prostate cancer: Evidence, controversies and future perspectives. Int I Urol 2009;16:458-64. http://dx.doi.org/10.1111/i.1442-2042.2009.02293.x

4. Ito K, Yamamoto $T$, Kubota $Y$, et al. Usefulness of age-specific reference range of prostate-specific antigen for Japanese men older than 60 years in mass screening for prostate cancer. Urology 2000;56:278-82. http://dx.doi.org/10.1016/S0090-4295(00)00613-0

5. Hugosson J, Carlsson S, Aus G, et al. Mortality results from the Goteborg randomized population-based prostate cancer screening trial. Lancet Oncol 2010;11:725-32. http://dx.doi.org/10.1016/S14702045(10)70146-7

6. Schröder FH, Hugosson J, Roobol MJ, et al. Screening and prostate cancer mortality in a randomized European study. New Engl J Med 2009;360:1320-8. http://dx.doi.org/10.1056/NEJMoa0810084

7. Roobol MJ, Kerkhof PSchröder FH, et al. Prostate cancer mortality reduction by prostate-specific antigenbased screening adjusted for nonattendance and contamination in the European Randomized Study of Screening for Prostate Cancer (ERSPC). Eur Urol 2009;56:584-91. http://dx.doi.org/10.1016/i. eururo.2009.07.018

8. Orsted DD, Nordestgaard BG, Jensen GB, et al. Prostate-specific antigen and long-term prediction of prostate cancer incidence and mortality in the general population. Eur Urol 2012;61:865-74. http:// dx.doi.org/10.1016/i.eururo.2011.11.007

9. Van Leeuwen PJ, Roobol MJ, Kranse R, et al. Towards an optimal interval for prostate cancer screening. Eur Urol 2012;61:171-6. http://dx.doi.org/10.1016/i.eururo.2011.08.002

10. Roobol MJ, van Vugt HA, Loeb $S$, et al. Prostate cancer risk: The role of prostate volume and digital rectal examination in the ERSPC risk calculators. Eur Urol 2012;61:577-83. http://dx.doi.org/10.1016/i. eururo.2011.11.012

11. Andriole GL, Crawford ED, Grubb RL 3rd, et al. Prostate cancer screening in the randomized Prostate, Lung, Colorectal, and Ovarian Cancer Screening Trial: Mortality results after 13 years of follow-up. J Natt Cancer Inst 2012;104:125-32. http://dx.doi.org/10.1093/inci/dir500

12. Chou R, Croswell JM, Dana T, et al. Screening for prostate cancer: A review of the evidence for the U.S. preventive services task force. Ann Intern Med 201 1;155:762-71. http://dx.doi.org/10.7326/00034819-155-11-201112060-00375

13. Jonsson H, HolPStröm B, Duffy SW, et al. Uptake of prostate-specific antigen testing for early prostate cancer detection in Sweden. Int J Cancer 2011;129:1881-8. http://dx.doi.org/10.1002/ijc.25846

14. Drummond FJ, Carsin AE, Sharp L, et al. Trends in prostate specific antigen testing in Ireland: Lessons from a country without guidelines. Ir J Med Sci 2010;179:43-9. http://dx.doi.org/10.1007/s1 1845009-0376-7

15. Smith DP, Supramaniam R, Marshall VR, et al. Prostate cancer and prostate-specific antigen testing in New South Wales. Med J Aust 2008;189:315-8.

16. Kubota $Y$, Ito $K$, Imai $K$, et al. Effectiveness of mass screening for the prognosis of prostate cancer patients in Japanese communities. Prostate 2002;50:262-9. http://dx.doi.org/10.1002/pros.10057

17. Aus $G$, Bergdahl $S$, Lodding $P$, et al. Prostate cancer screening decrease the absolute risk of being diagnosed with advanced prostate cancer: Results from a prospective, population-based randomized control trial. Eur Urol 2007;51:659-64. http://dx.doi.org/10.1016/i.eururo.2006.07.012

18. Schröder FH, Hugosson J, Carlsson S, et al. Screening for prostate c ancer decreases the risk of developing metastatic disease: Findings from the European Randomized Study of Screening for Prostate Cancer (ERSPC). Eur Urol 2012;62:745-52. http://dx.doi.org/10.1016/j.eururo.2012.05.068

19. Roemeling S, Roobol MJ, Gosselaar C, et al. Biochemical progression rates in the screen arm compared to the control arm of the Rotterdam Section of the European Randomized Study of Screening for Prostate Cancer (ERSPC). Prostate 2006;66:1076-81. http://dx.doi.org/10.1002/pros.20391

20. Raaijmakers R, Kirkels WJ, Roobol MJ, et al. Complication rates and risk factors of 5802 transrectal ultrasound-guided sextant biopsies of the prostate within a population-based screening program. Urology 2002;60:826-30. http://dx.doi.org/10.1016/S0090-4295(02)01958-1

21. Johansson E, Bill-Axelson A, Holmberg L, et al. Time, symptom burden, androgen deprivation, and selfassessed quality of life after radical prostatectomy or watchful waiting: The Randomized Scandinavian Prostate Cancer Group Study Number 4 (SPCG-4) clinical trial. Eur Urol 2009;55:422-30. http://dx.doi. org/10.1016/i.eururo.2008.08.054 
Teishima et al.

22. Thong PS, Mols F, Kil PJ, et al. Prostate cancer survivors who would be eligible for active surveillance but were either treated with radiotherapy or managed expectantly: Comparisons on long-term quality of life and symptom burden. BJU Int 2010;105:625-8. http://dx.doi.org/10.1111/i.1464410X.2009.08815.x
Correspondence: Dr. Jun Teishima, Department of Urology, Integrated Health Sciences, Institute of Biomedical and Health Sciences, Hiroshima University, 1-2-3 Kasumi, Minamiku, Hiroshima 734-8551, Japan; teishimaj@yahoo.co.jp 